\title{
Convolutional neural network vs bag of features for bambara groundnut leaf disease recognition
}

\author{
Hafizatul Hanin Hamzah ${ }^{1}$, Nurbaity Sabri ${ }^{2}$, Zaidah Ibrahim ${ }^{3}$, Dino Isa ${ }^{4}$ \\ ${ }^{1,3}$ Faculty of Computer and Mathematical Sciences, Universiti Teknologi MARA, Shah Alam, Selangor, Malaysia \\ ${ }^{2}$ Faculty of Computer and Mathematical Sciences, Universiti Teknologi MARA, Campus Jasin, Melaka, Malaysia \\ ${ }^{4}$ CONNECT Initiative, Crops for the Future, Jalan Broga, Semenyih, Selangor, Malaysia
}

\begin{tabular}{l} 
Article Info \\
\hline Article history: \\
Received Sep 2, 2018 \\
Revised Nov 30, 2018 \\
Accepted Dec 12, 2018 \\
\hline Keywords: \\
BoF \\
CNN \\
Leaf disease recognition \\
SURF \\
SVM
\end{tabular}

\begin{abstract}
This paper investigates bambara groundnut leaf disease recognition using two popular techniques known as Convolutional Neural Network (CNN) and Bag of Features (BOF) with Speeded-up Robust Feature (SURF) and Support Vector Machine (SVM) classifier. Leaf disease recognition has attracted many researchers because the outcome is useful for farmers. One of the crops that provide high income for farmers is bambara groundnut but the leaves are easily infected with diseases especially after the rain. This could affect the crop productivity. Thus, automatic disease recognition is crucial. A new dataset that consists of 400 images of the infected and non-infected leaves of bambara groundnut has been constructed. The experimental results indicate that both of these techniques produce excellent leaf disease recognition accuracy.
\end{abstract}

Copyright $@ 2019$ Institute of Advanced Engineering and Science. All rights reserved.

\section{Corresponding Author:}

Hafizatul Hanin Hamzah

Faculty of Computer and Mathematical Sciences,

Universiti Teknologi MARA,

Shah Alam, Selangor, Malaysia

Email: haninhamzah94@gmail.com

\section{INTRODUCTION}

Bambara groundnut, or the scientific name Vigna subterranea (L) Verdc. is originally planted in the African continent and has been cultivated in tropical Africa for centuries [1]. It has been planted in Malaysia due to similar weather condition but one of the challenges planting it here is that it can easily be infected with leaf diseases after heavy rains [1]. In order to minimize the leaf disease that induced damage during the growth of bambara groundnut, harvest and post-harvest processing, as well as maximize productivity and ensure agricultural sustainability, automatic leaf disease recognition is highly important [2]. The existing method for leaf plant disease recognition is simply applying the naked eye observation by experts [3]. In doing so, a large team of experts as well as continuous monitoring of plant is required, which incur costs for large farms [3]. Plant disease recognition by visual way is more laborious and time consuming and at the same time, less accurate and can be done only in limited areas [4].

In order to adapt to this fast changing environment, appropriate and timely plant leaf disease recognition is crucial. However, most plant leaf diseases generate some kind of manifestation in the visible spectrum, so the naked eye examination of a trained professional is the prime technique adopted in practice for plant disease recognition [5]. An automated plant leaf disease recognition system could be of great help for amateurs in the gardening process and also trained professionals as a verification system in disease diagnostics [6]. Various features and classifiers have been investigated to recognize plant diseases automatically [7]-[10]. Colour features and Back-Propagation Neural Network (BPNN) have been used for cotton and groundnut diseases classification [7]. Shape and colour features with Support Vector Machine (SVM) classifier have been utilized to classify rice-plant diseases [8]. SVM has also been used to classify 
cotton leaf spot disease in [9]. A comparative study has been performed among various texture features namely Local Binary Pattern (LBP) and Gray Level Co-occurrence Matrix (GLCM) and classifiers that are Probabilistic Neural Network (PNN), BPNN, SVM and Random Forest (RF) to classify diseases in grapes and the results indicate that GLCM with RF achieve the best recognition results [11]. Convolutional Neural Network $(\mathrm{CNN})$ is getting popular in object recognition problems such as leaf recognition [13-14], fruit recognition [15-16], character recognition [17], vehicle recognition [18] and palm oil fresh fruit bunch ripeness grading recognition [19]. Plant disease classification based on CNN produce outstanding accuracy results [20]. BoF, one of the many machine learning techniques, has also shown good performance in object recognition [21-22]. Due to promising results produced by $\mathrm{BoF}$ and $\mathrm{CNN}$, this research plans to investigate their performances in recognizing bambara groundnut leaf disease.

\section{RESEARCH METHOD}

\subsection{Convolutional Neural Network $(\mathrm{CNN})$}

The architecture of CNN is structured as a series of layers, that consists of three layers which are convolve layer, pooling layer and Rectified Linear unit (ReLu) [16]. Convolve layer extracts features of an image using filter and image patch that strides over the input image. ReLu layer replaces all negative pixel values in the feature map with zero while pooling layer allows the feature map to be down-sampled after ReLu layer to reduce the dimensionality. Max-pooling computes the maximum local of feature map. Neighboring pooling takes input from feature maps that are shifted or stride by more than one rows or columns. Figure 1 shows the architecture of a CNN [23].

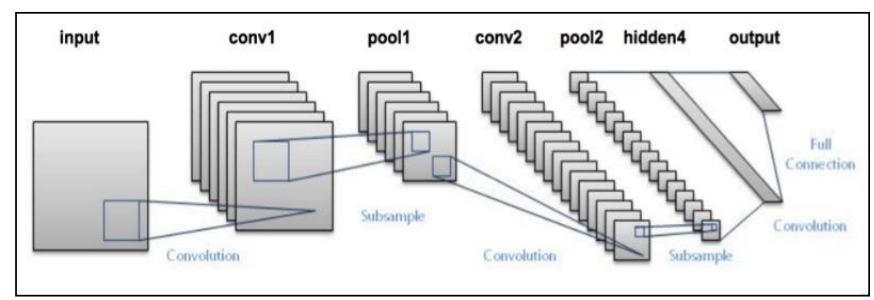

Figure 1. The architecture of CNN [26]

\subsection{Bag of Features $(\mathrm{BoF})$}

One method that represents images as orderless collections of local features is called Bag of Features (BoF) [22]. In this project, Speeded up Robust Features (SURF) has been used in BoF because the performance of this feature is excellent and only require low computational cost [24]. It is a descriptor that is based on Hessian matrix measures and an image detector. For a descriptor which uses only 64 dimensions leading to quick feature extraction, and it also uses a 2D Haar wavelet transform [24].The two common perspectives for the $\mathrm{BoF}$ image representation explanation which the first one is the by analogy from the Bag of Words representation. One represents a document that normalizes histogram of word counts with Bag of Words, [25]. Figure 2 shows the process for BoF image representation.

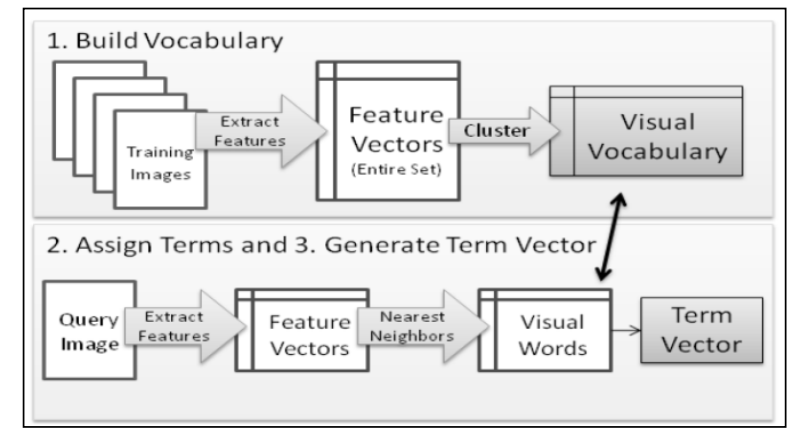

Figure 2. Process for BoF image representation [22] 


\section{RESULTS AND ANALYSIS}

\subsection{The Dataset}

A new dataset of the bambara groundnut leaf images has been constructed that consists of 200 images of the non-infected leaves and 200 images of leaves with diseases. They were captured from a farm in Semenyih, Selangor using a mobile phone. Some sample images of bambara groundnut with and without diseases are illustrated in Figure 3 and Figure 4.

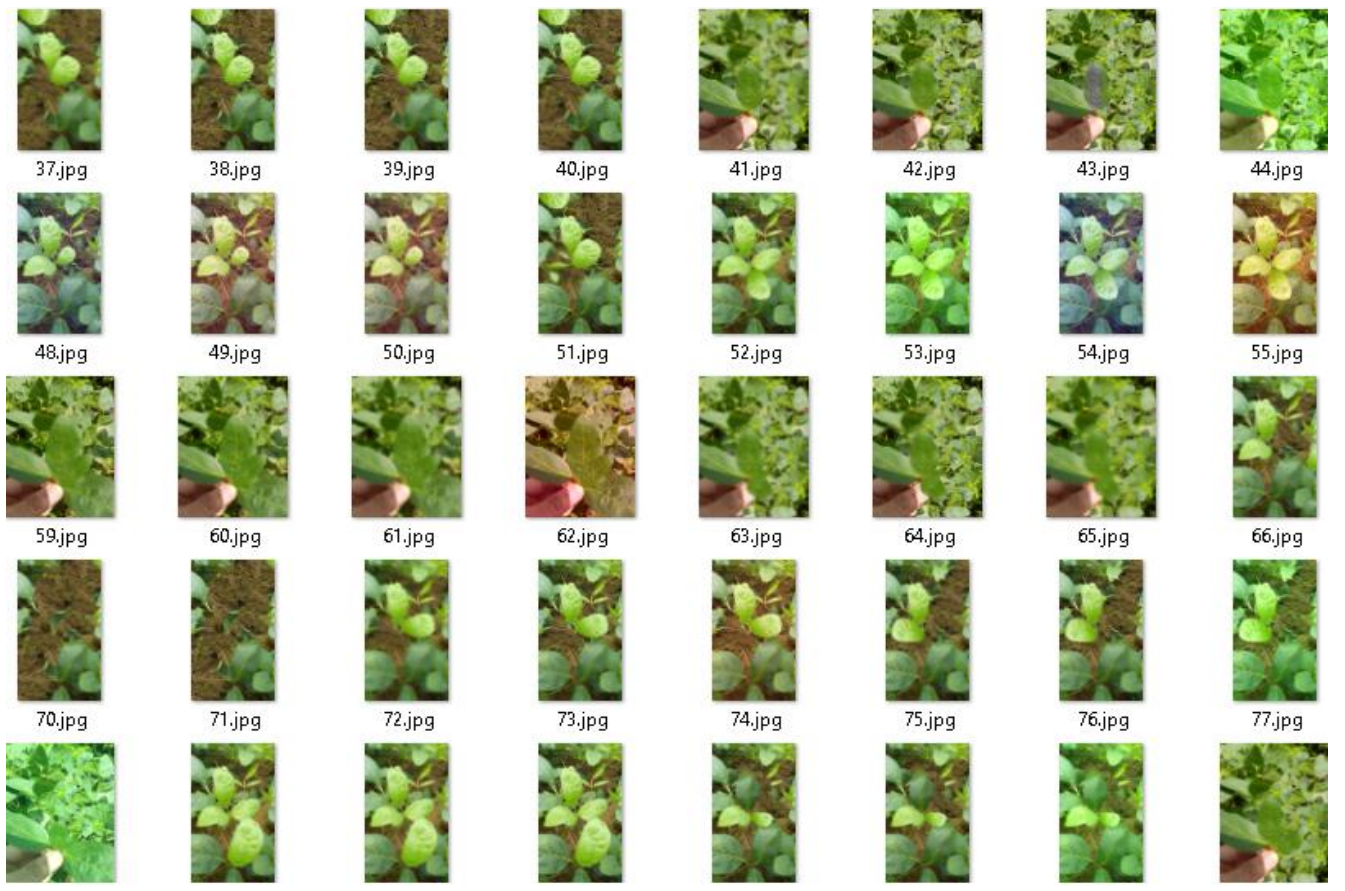

Figure 3. Some sample images of bambara groundnut without leaf diseases

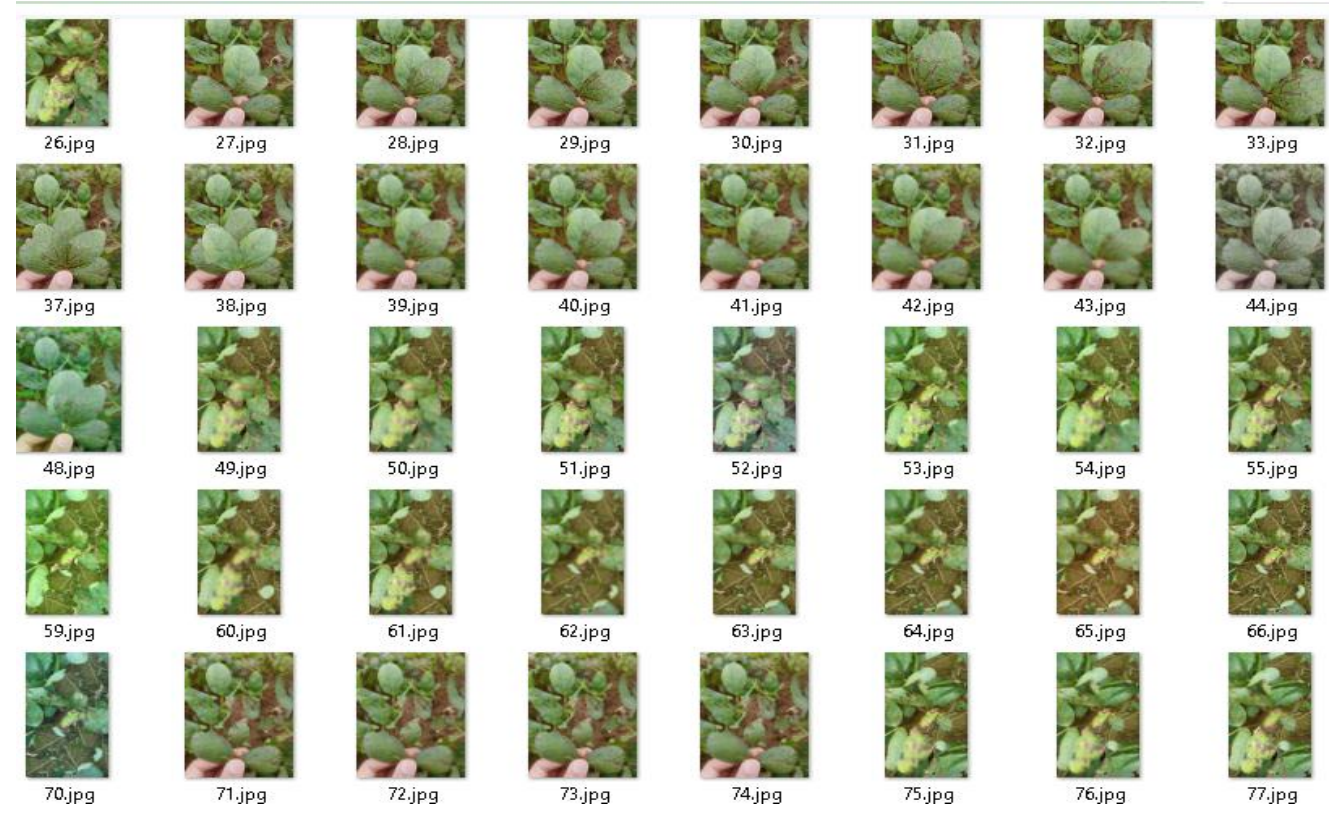

Figure 4. Some sample images of bambara groundnut with leaf diseases 


\subsection{CNN}

A stack of CNN consist of convolve layer, pooling layer and ReLu layer while additional stack of layers can be added to improve the performance. $\mathrm{CNN}$ takes color images and the features are automatically extracted by the convolve layers. The size of filters in the convolve layer and the value of stride in the pooling layer represent the number of columns to be skipped for the sliding window through the image. These values can be changed as they can affect the result of the recognition performance. Besides that, the values of epochs represent the number of iteration for the training process and initial learning rate that represent the value of the weight to be adjusted during the training process, can be changed to view their effect to the recognition rate. The image size required for basic CNN is 224 x 224 pixels. Experimental results were conducted on the combination of these values and the results are shown in Table 1. The first column represents the size of the filter and the number of filters in the convolve layer. By referring to Table 1 , we can see that a $100 \%$ accuracy is achieved with $[5,20]$ in the first convolve layer and $[3,32]$ in the second convolve layer, and Figure 5 shows the results of this training and validation processes.

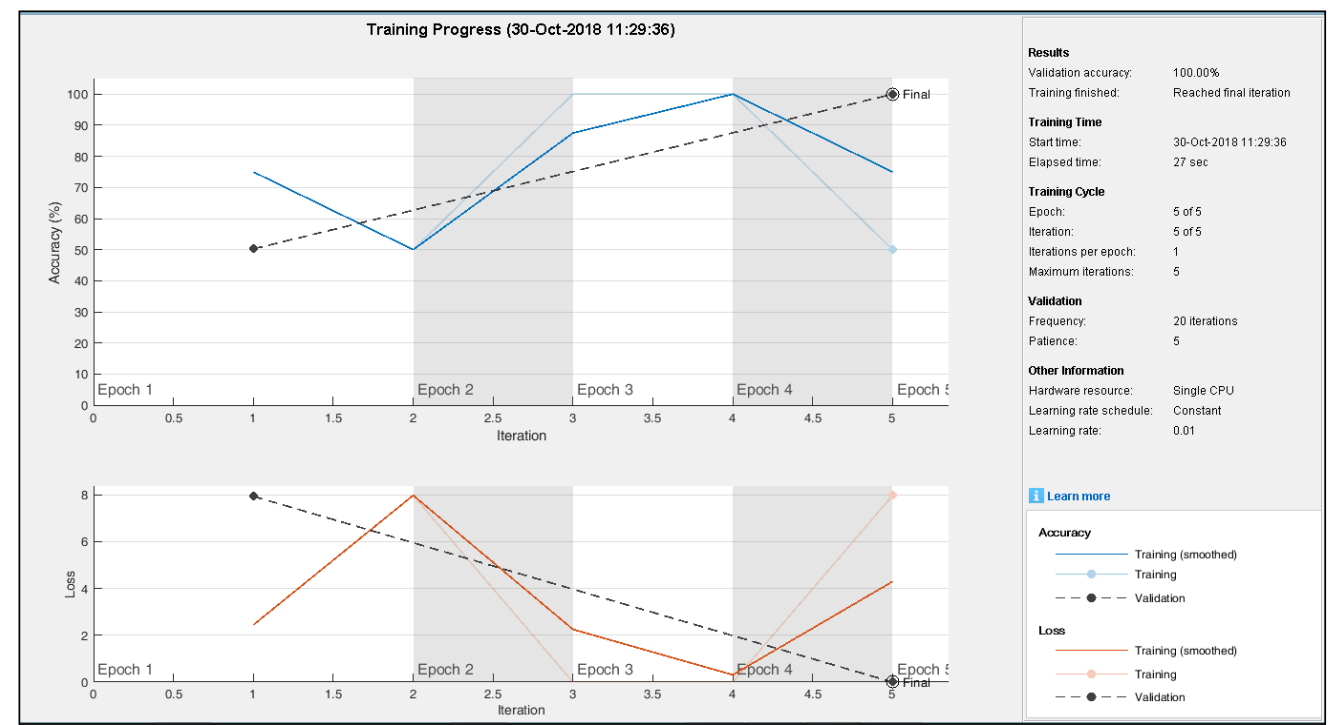

Figure 5. The result of CNN with [5,20] in the first convolve layer and [3,32] in the second convolve layer Table 1. Experimental Results on Parameter Tuning for Basic CNN

\begin{tabular}{ccccc}
\hline No of Stack of Layers & Convolve Layer & Pooling layer and Stride & Accuracy (\%) & Total Time/s \\
\hline 1 & {$[3,16]$} & 3 & 78.82 & $30 \mathrm{sec}$ \\
2 & {$[5,20]$} & 3 & 83.59 & $28 \mathrm{sec}$ \\
\multirow{2}{*}{3} & {$[3,16][3,32]$} & 3 & 91.79 & $27 \mathrm{sec}$ \\
& {$[5,20][3,32]$} & 2 & 100.00 & $27 \mathrm{sec}$ \\
& {$[5,20][3,32][3,32]$} & 2 & 75.90 & $41 \mathrm{sec}$ \\
& {$[5,20][3,32][3,16]$} & 2 & 74.87 & $34 \mathrm{sec}$ \\
\hline
\end{tabular}

By looking at Table 1, we can see that as the number of layers increases, the accuracy is also increased. But when the number of layers is more than 3, the accuracy begins to drop. This means that two stacks of layers plus 1 classification layer produce the best accuracy for bambara groundnut lead disease recognition.

\subsection{Bag of Features (BoF)}

The size of an image used for BoF is 227 x 227 pixels and the accuracy produced is $100 \%$. Figure 6 shows the result of visual words occurrence produced by BoF for our dataset. Speeded-Up Robust Feature (SURF) and Support Vector Machine (SVM) is being utilized in the BoF. 


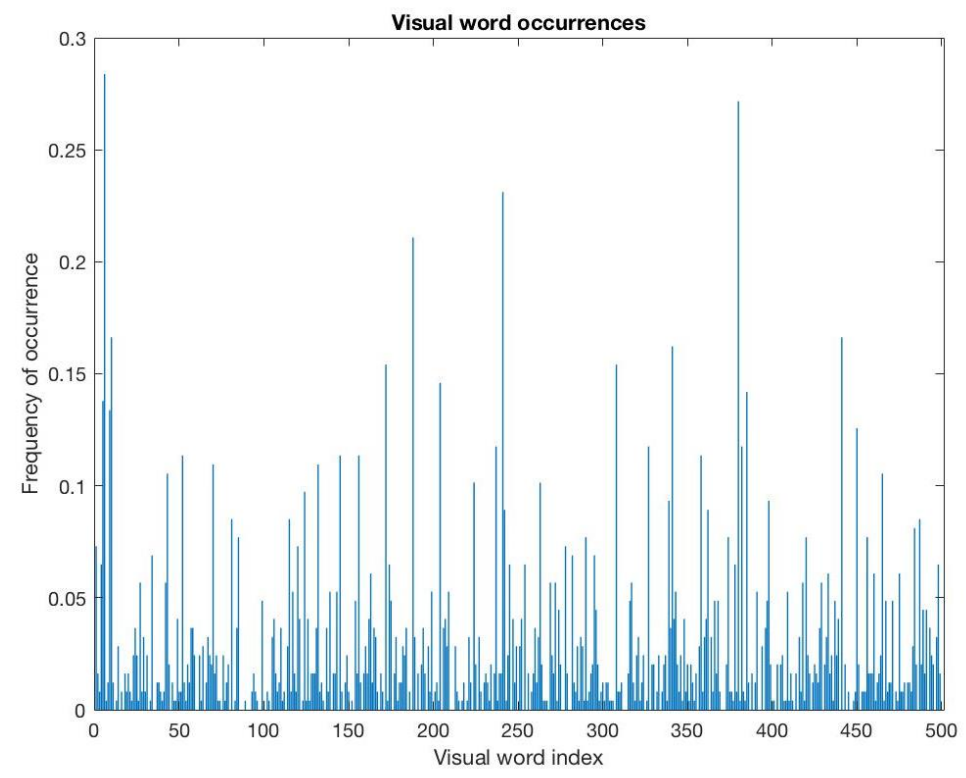

Figure 6. Visual word occurrences result

Table 2 shows an overview of the accuracy performance of CNN compared to BoF based on our bambara groundnut leaf dataset. By looking at Table 2, we can see that BoF is better than basic CNN but it took a longer time to achieve this result. This is because extracting of the SURF features is longer compared to the time to extract the low-level and middle level features by the CNN.

Table 2. The Performance Overview for Basic CNN and BoF for Bambara Dataset

\begin{tabular}{lcc}
\hline Model & Basic CNN & BoF \\
\hline Validation accuracy & 100 & 100 \\
Elapsed Time (s) & 27 & 31 \\
\hline
\end{tabular}

\section{CONCLUSION}

In this paper, a comparison between $\mathrm{CNN}$ and $\mathrm{BoF}$ was performed with respect to accuracy and elapsed time. The experiment results show that BoF achieved the same accuracy rate as CNN which is $100 \%$. However, BoF requires a higher elapsed time due to the large number of SURF features required to be extracted. Although the number of layers affects the accuracy performance, the complexity of the CNN architecture does not guarantee a better result. The experimental results in this research indicate that two stacks of layers produce better accuracy compared to three stacks of layers. The use of CNN is recommended for leaf disease recognition if the processing time is not an issue. For future work, more deep learning models and publicly available datasets will be tested.

\section{ACKNOWLEDGEMENTS}

The authors would like to thank Faculty of Computer and Mathematical Sciences, Universiti Teknologi MARA, Shah Alam, Selangor, for sponsoring this research.

\section{REFERENCES}

[1] Azman, R., Mayes, S., \& Lu, C. Nutritional Profile Of Bambara Groundnut And Its Potential For Food Product Development In Malaysia. Model International Underutilised Legume Research And Breeding Journal., 24(4), . (2016) 429. Http://Dx.Doi.Org/10.4314/Acsj.V24i4.9).

[2] Fang, Y., \& Ramasamy, R. Current And Prospective Methods For Plant Disease Detection. Biosensors, 5(3), Http://Dx.Doi.Org/10.3390/Bios5030537 (2016) 537-561. 
[3] Singh, V., \& Misra, A. Detection Of Plant Leaf Diseases Using Image Segmentation And Soft Computing Techniques. Information Processing In Agriculture, 4(1Http://Dx.Doi.Org/10.1016/J.Inpa.2016.10.005 (2017),41-49

[4] Shetye, H., Rane, T., Pawar, T., \& Dandwate, P. An Analysis Of Methodologies For Leaf Disease Detection Techniques. International Journal Of Research In Advent Technology, Vol.4, No.2, February 2016, Vol.4(No.2), 5358. Http://Dx.Doi.Org/E-Issn:. (2016). 2321-9637

[5] Ibrahim, Z., Sabri, N., \& Mangshor, N. N. A Leaf Recognition using Texture Features for Herbal Plant Identification, IJEECS, vol. 9, . (2018). no. 1, 152-156.

[6] Sladojevic, S., Arsenovic, M., Anderla, A., Culibrk, D., \& Stefanovic, D.. Deep Neural Networks Based Recognition Of Plant Diseases By Leaf Image Classification. Computational Intelligence And Neuroscience, 2016. (2016)

[7] Pilli, S. K., Nallathambi, B. George, S. J. and Diwanji, V., "eAGROBOT- A Robot for Early Crop Disease Detection using Image Processing", IEEE $2^{\text {nd }}$ International Conference on Electronics and Communication System (ICECS), 2015.

[8] Yao, Q. et. al. "Application of support vector machine for detecting rice diseases using shape and colour texture features", International Conference on Engineering and Computation, 2009, pp. 79-83.

[9] Patil, S. P. and Zambre, R. S. "Classification of Cotton Leaf Spot Disease Using Support Vector Machine”, Journal of Engineering Research and Applications, Vol. 4, Issue 5, Version 1, 2010, pp. 10-20.

[10] Fujita, E., Kawasaki, Y., Uga, H., Kagiwada, S. and Iyatomi, H., "Basic investigation on a robust and practical plant diagnostic system", $15^{\text {th }}$ IEEE International Conference on Machine Learning and Applications, 2016, pp. 989-992.

[11] Sandika, B., Avil, S., Sanat, S. and Srinivasu, P., "Random Forest based Classification of Diseases in Grapes from Images Captured in Uncontrolled Environments", IEEE International Conference on Signal Processing, 2016, pp. 1775-1780.

[12] Sasano, S., Han, X. and Chen, Y., "Food Recognition by Combined Bags of Color Features and Texture Features", IEEE $9^{\text {th }}$ International Congress on Image and Signal Processing, BioMedical Engineering and Informatics (CISPBMEI), 2016, pp. 815-819.

[13] Zaidah Ibrahim, Nurbaity Sabri and Dino Isa, "Multi-maxpooling Convolutional Neural Network for Medicinal Herb Leaf Recognition", Proceedings of the 6th IIAE International Conference on Intelligent Systems and Image Processing, Shimane, Japan, September 2018 , pp. 327-331.

[14] Nurbaity Sabri, Zalilah Abd Aziz, Zaidah Ibrahim, Muhammad Akmal Rasydan bin Mohd Rosni and Abdul Hafiz bin Abd Ghapul, "Comparing Convolutional Neural Network Models for Leaf Recognition", International Journal of Engineering Technology, 7 (3.15) (2018) pp. 141-144.

[15] Nur Azida Muhammad, Amelina Ab Nasir, Zaidah Ibrahim and Nurbaity Sabri, "Evaluation of CNN, Alexnet and GoogleNet for Fruit Recognition", Indonesian Journal of Electrical Engineering and Computer Science (IJEESC), Vol. 12, No. 2, November 2018, pp. 468-475.

[16] Hou, L., Wu, Q., Sun, Q., Yang, H., \& Li, P. Fruit recognition based on convolution neural network. 2016 12th International Conference on Natural Computation, Fuzzy Systems and Knowledge Discovery, ICNC-FSKD 2016, 18-22.

[17] Muhaafidz Md Saufi, Mohd Afiq Zamanhuri, Norasiah Mohammad and Zaidah Ibrahim "Deep Learning for Roman Handwritten Character Recognition", International Journal of Electrical Engineering and Computer Science, Vol. 12, No. 2, November 2018, pp. 455-460.

[18] Raja Durratun Safiyah, Zaid Abdul Rahim, Syamsul Syafiq, Zaidah Ibrahim and Nurbaity Sabri "Performance Evaluation for Vision-Based Vehicle Classification Using Convolutional Neural Network", International Journal of Engineering \& Technology, 7 (3.15), (2018), pp. 86-40.

[19] Zaidah Ibrahim, Nurbaity Sabri and Dino Isa, "Palm Oil Fresh Fruit Bunch Ripeness Grading Recognition Using Convolutional Neural Network", Journal of Telecommunication, Electronic \& Computer Engineering, Vol 9, No. 32, 2018, pp. 109-113.

[20] Gupta, D., Jain, K., Jain, A., \& Analytics Vidhya Content Team. Architecture of Convolutional Neural Networks (CNNs) demystified. (2017, June 29).

[21] Schmid, Cordelia. "Bag-of-features for category classification." ENS/INRIA Visual Recognition and Machine Learning Summer School Lecture 25-29 July (2011).

[22] O'Hara, Stephen, and Bruce A. Draper. "Introduction to the bag of features paradigm for image classification and retrieval." arXiv preprint arXiv:1101.3354 (2011).

[23] Muhammad,A., Nasir A., Ibrahim, Z, Sabri, N., Evaluation of CNN, Alexnet and GoogleNet for Fruit Recognition. Indonesian Journal of Electrical Engineering and Computer Science 2018, pp. 468 475

[24] Ahmad, K., Khan, R., Ahmad, N. and Khan, J. "Evaluation of SIFT and SURF using Bag of Words Model on a Very Large Dataset,” Sindh Univ. Res. Jour. (Sci. Ser.) Vol.45 (3) 492-495 (2013).

[25] S. H. Lee, C. S. Chan, P. Wilkin, and P. Remagnino, "Deep-plant: Plant identification with convolutional neural networks,” IEEE International Conference on Image Processing (ICIP), 2015. 


\section{BIOGRAPHIES OF AUTHORS}

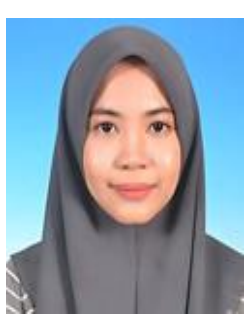

Hafizatul Hanin Hamzah is a Master's student at the Faculty of Computer and Mathematical Sciences, Universiti Teknologi MARA, Shah Alam, Selangor, Malaysia, where she is continuing her education in the field of Computer Science in Web Technology. Her area of interests are image processing, image recognition, and multimedia computing.

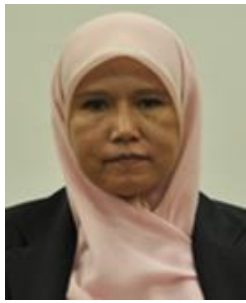

Zaidah Ibrahim is an Associate Professor at the Faculty of Computer and Mathematical Sciences, Universiti Teknologi MARA, Shah Alam, Selangor, Malaysia. She has been teaching courses related to Artificial Intelligence for more than ten years. She is actively involved in research and publications under Digital Image, Audio and Speech Technology (DIAST) research interest group that includes computer vision and intelligent systems.

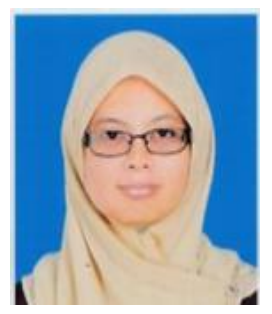

Nurbaity Sabri is a lecturer at the Faculty of Computer and Mathematical Sciences, Universiti Teknologi MARA, Jasin, Melaka. She teaches programming language and image processing. She is a member of Digital Image, Audio and Speech Technology (DlAST) research group and currently participating in various researches related to image processing. Recently, she has published papers and co-authored in international conferences and journals. Her research interests include image processing, computer vision and pattern recognition.

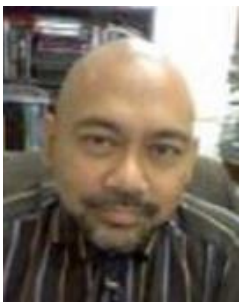

Prof. Dino Isa is a professor of Intelligent Systems at University of Nottingham Malaysia Campus, Semenyih, Selangor, since 2018, and has published more than 100 papers in his field of research. He has been appointed as Director, CONNECT Initiative, Crops for the Future, involving in agriculturerelated projects using machine learning to infer the level of profits available to farmers when underutilized crops are grown and its derivatives are processed on a commercial scale. He is currently the chief consultant to Tiger Solutions Sdn. Bhd., an integrated oil and gas technology company interested in using machine learning to predict failure in oil and gas pipelines. 